\title{
MATHEMATICAL ANALYSIS AND NUMERICAL SIMULATION OF A REYNOLDS-KOITER MODEL FOR THE ELASTOHYDRODYNAMIC JOURNAL-BEARING DEVICE*
}

\author{
IÑigo Arregui ${ }^{1}$, J. Jesús Cendán ${ }^{1}$ And Carlos VÁzquez ${ }^{1}$
}

\begin{abstract}
The aim of this work is to deduce the existence of solution of a coupled problem arising in elastohydrodynamic lubrication. The lubricant pressure and concentration are modelled by Reynolds equation, jointly with the free-boundary Elrod-Adams model in order to take into account cavitation phenomena. The bearing deformation is solution of Koiter model for thin shells. The existence of solution to the variational problem presents some difficulties: the coupled character of the equations, the nonlinear multivalued operator associated to cavitation and the fact of writing the elastic and hydrodynamic equations on two different domains. In a first step, we regularize the Heaviside operator. Additional difficulty related to the different domains is circumvented by means of prolongation and restriction operators, arriving to a regularized coupled problem. This one is decoupled into elastic and hydrodynamic parts, and we prove the existence of a fixed point for the global operator. Estimations obtained for the regularized problem allow us to prove the existence of solution to the original one. Finally, a numerical method is proposed in order to simulate a real journal-bearing device and illustrate the qualitative and quantitative properties of the solution.
\end{abstract}

Mathematics Subject Classification. 35R35, 74K25, 76D03, 76D08.

Received: June 21, 2001. Revised: December 17, 2001.

\section{INTRODUCTION}

The journal-bearing is a lubricated device that takes part in thermal engines, compressors, turbomachinery and gear boxes, for example. Briefly, it consists of a cylindrical journal which rotates inside a fixed cylindrical bearing. The thin gap between them is filled with a lubricant which dumps heating and friction (see Fig. 1). In this device, as in other lubricated devices, it is very important to consider a well suited mathematical model in order to predict the behaviour of the lubricant pressure distribution, the formation of air bubbles near the contact region as well as the gap profile which balances the hydrodynamic load and a given load vector which is, eventually, imposed on the device. These previous facts have widely motivated an increasing study of thin fluid film displacement as well as its coupled behaviour with the elastic deformation of the boundary surfaces, concerning with the modelling, mathematical analysis and numerical simulation aspects $[1,2,4,6,14,17,19-21]$.

\footnotetext{
Keywords and phrases. Koiter model, Reynolds equation, free boundary problems, fixed point techniques.

* The authors thank MCYT (project BFM2001-3261-C02-02), Xunta de Galicia (project XUGA 32201B97) and Universidade da Coruña, for the financial support of this work.

1 Departamento de Matemáticas, Facultad de Informática, University of La Coruña, Campus de Elviña, s/n, 15071 La Coruña, Spain. e-mail: arregui@udc.es, suceve@udc.es, carlosv@udc.es
} 

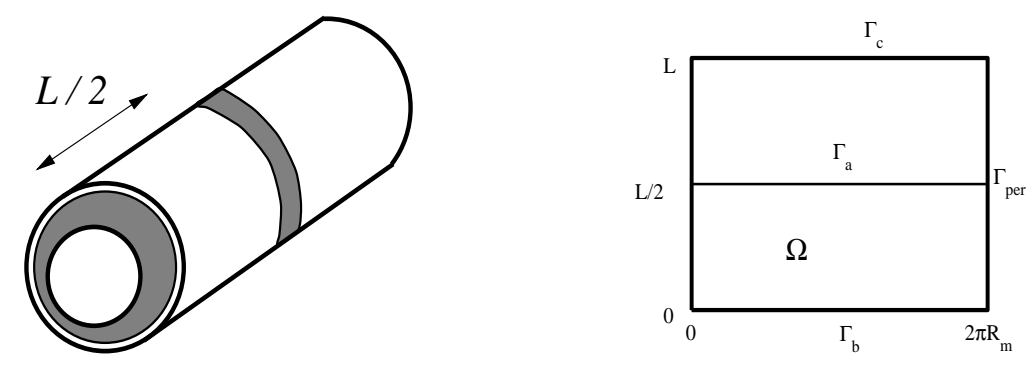

FiguRE 1. Journal bearing device.

In a previous work [2], we have presented a coupled model for simulating an elastohydrodynamic journalbearing device, the mathematical analysis being still an open problem. The small thickness of the gap occupied by the fluid allows the introduction of the bidimensional Reynolds equation to approximate the three dimensional Stokes equation, as it is rigurously justified [3]. In this way, we are considering a newtonian, incompressible, isothermal and isoviscous lubricant. Indeed, following Reynolds approach, we assume that the pressure is constant through the thickness of the film. Moreover, the converging-diverging geometry of the gap gives place to pressure values below saturation pressure, so that air bubbles appear (cavitation). Therefore, as Reynolds equation is no longer valid in the bubble region, a cavitation model must be introduced [4]. As in previous works, the more realistic one (Elrod-Adams) introduces a saturation function as additional unknown.

Nevertheless, in some industrial cases the high pressure values can give place to elastic deformations in the bearing. In [2], we assume that the bearing is thin enough to approximate its elastic behaviour by means of a Koiter shell model (the bearing is thin, but not necessarily shallow). An important difference between the present paper and [2] concerns with boundary conditions. Thus, we replace the Koiter formulation posed over half of the device with a symmetry condition by a formulation on the whole device with clamped boundary conditions at both ends. The coupled aspect of the model results from the fact that the lubricant pressure is a normal force in the elastic Koiter model and the normal displacement of the bearing is a contribution to the gap in the hydrodynamic Reynolds equation.

This paper is mainly devoted to the mathematical analysis of the new model, which differs from the one used in [2] as explained before. Main difficulties arise from the nonlinearities and the presence of free boundaries due to cavitation. In order to prove the existence of solution to the coupled problem, we have followed previous works $[6,14]$, where the solution is the fixed point of a global operator. In the present case, the existence of a fixed point results to be some more difficult to state, due to the fact that elastic and hydrodynamic equations are not written in the same domain. An algorithm which essentialy adapts the one developed in [2] to the new coupled model provides some numerical results.

In Section 1, the variational equations of the coupled model and the regularized problem are posed. In Section 2, we prove the existence of solution to the regularized problem as a fixed point of a global operator, which consists of the composition of four operators related to Koiter model, cavitation model for Reynolds equation, and both restriction and prolongation operators in order to pass from one domain to another. In Section 3, the existence of solution to the original coupled problem is proved as the limit of a sequence of solutions to regularized problems. Finally, in Sections 4 and 5 we describe the approximation techniques we have used in the simulations and some numerical results. 


\section{The Reynolds-Koiter Coupled problem}

\subsection{The continuous hydrodynamic problem}

In this section, the mathematical coupled problem for the previously described elastic journal bearing device is posed. Let us consider the sets

$$
\Omega=\left(0,2 \pi R_{m}\right) \times(0, L / 2), \quad \widetilde{\Omega}=\left(0,2 \pi R_{m}\right) \times(0, L)
$$

where $L$ is the length of the device and $R_{m}=0.5\left(R_{b}+R_{j}\right)$ represents the mean radius, $R_{b}$ and $R_{j}$ being the bearing and the journal radii, respectively. Thus, the set $\widetilde{\Omega}$ corresponds to the usual cylindrical parametrization domain for the mean cylinder between the journal and the bearing. The same holds for the set $\Omega$, which is associated to half of the device. In terms of $\Omega, \widetilde{\Omega}$ and the pressure we define the new sets:

$$
\begin{array}{ll}
\Omega^{+}=\left\{\left(x_{1}, x_{2}\right) \in \Omega / p\left(x_{1}, x_{2}\right)>0\right\} & \Gamma_{a}=\left\{\left(x_{1}, x_{2}\right) \in \partial \Omega / x_{2}=L / 2\right\} \\
\Omega_{0}=\left\{\left(x_{1}, x_{2}\right) \in \Omega / p\left(x_{1}, x_{2}\right)=0\right\} & \Gamma_{b}=\left\{\left(x_{1}, x_{2}\right) \in \partial \Omega / x_{2}=0\right\} \\
\Sigma=\partial \Omega^{+} \cap \partial \Omega_{0} & \Gamma_{\text {per }}=\left\{\left(x_{1}, x_{2}\right) \in \partial \Omega / x_{1}=0 \text { or } x_{1}=2 \pi R_{m}\right\} \\
& \Gamma_{c}=\left\{\left(x_{1}, x_{2}\right) \in \partial \widetilde{\Omega} / x_{2}=L\right\}
\end{array}
$$

where $p$ is the lubricant pressure, and $x_{1}$ and $x_{2}$ represent the angular and longitudinal coordinates. The sets $\Gamma_{b}$ and $\Gamma_{c}$ are the clamped boundaries of the bearing which are in contact with atmospheric pressure. The set $\Gamma_{a}$ represents the lubricant supply groove and the boundary $\Gamma_{\text {per }}$ constitutes a periodic boundary for the pressure, while it does not exist in the formulation of the elastic problem. Notice that the points $\left(0, x_{2}\right)$ and $\left(2 \pi R_{m}, x_{2}\right)$ are identified in the $3-D$ real device.

In the hydrodynamic part of the problem (thin film lubricant displacement), the physical data are the constant lubricant viscosity $\left(\nu_{f}\right)$, the supply pressure $\left(p_{a}\right)$ and the journal rotation velocity $(s)$. Moreover, in absence of elastic deformations, the gap between the journal and the bearing is commonly approached in the form:

$$
h_{r}\left(x_{1}\right)=c\left(1+\varrho \cos \frac{x_{1}}{R_{m}}\right)
$$

where the clearance $c$ is the difference between the bearing and the journal radii and $\varrho \in(0,1)$ represents the journal-bearing eccentricity (see [10] for details about the device). Moreover, let us denote:

$$
\underline{h_{r}}=\min _{x_{1} \in\left[0,2 \pi R_{m}\right)} h_{r}=c(1-\varrho) \quad \overline{h_{r}}=\max _{x_{1} \in\left[0,2 \pi R_{m}\right)} h_{r}=c(1+\varrho) .
$$

Nevertheless, in elastohydrodynamic regimes the lubricant pressure induces an elastic displacement of the surfaces in contact which modifies the gap. In the particular journal bearing device with rigid journal and elastic thin bearing, the Koiter model for shells seems to be the most appropriate way to take into account the elastic effects related to the cylindrical geometry. 
Thus, the Reynolds problem is posed in $\Omega$ as follows:

Find $(p, \theta, \vec{u})$ such that:

$$
\begin{array}{r}
\frac{\partial}{\partial x_{1}}\left[\left(h_{r}+u_{3}\right)^{3} \frac{\partial p}{\partial x_{1}}\right]+\frac{\partial}{\partial x_{2}}\left[\left(h_{r}+u_{3}\right)^{3} \frac{\partial p}{\partial x_{2}}\right]=12 \nu_{f} s \frac{\partial\left(h_{r}+u_{3}\right)}{\partial x_{1}}, \quad p>0, \quad \theta=1 \quad \text { in } \Omega^{+} \\
\frac{\partial}{\partial x_{1}}\left[\theta\left(h_{r}+u_{3}\right)\right]=0, \quad p=0, \quad 0 \leq \theta \leq 1, \quad \text { in } \Omega_{0} \\
\left(h_{r}+u_{3}\right)^{3} \frac{\partial p}{\partial n}=12 \nu_{f} s(1-\theta)\left(h_{r}+u_{3}\right) \cos (\vec{n}, \vec{\imath}), \quad p=0 \quad \text { on } \Sigma \\
p=p_{a} \quad \text { on } \Gamma_{a} \\
p=0 \quad \text { on } \Gamma_{b} \\
p\left(0, x_{2}\right)=p\left(2 \pi R_{m}, x_{2}\right) \quad \text { if } 0<x_{2}<L / 2
\end{array}
$$

where $\vec{\imath}$ is the unit vector in $O x_{1}$ direction and $\vec{n}$ is the unit normal vector to $\Sigma$ pointing towards $\Omega_{0}$. In the previous equations, the unknowns $p, \theta$ and $\vec{u}=\left(u_{1}, u_{2}, u_{3}\right)$ represent the fluid pressure, the saturation variable in the Elrod-Adams model for cavitation (see [4] for details about cavitation models) and the bearing displacement generated by the lubricant pressure, respectively. Thus, conditions (1.3) correspond to Reynolds equation and are verified in the fluid region, equation (1.4) corresponds to Elrod-Adams model for the cavitation region, conditions (1.5) are imposed on the free boundary $\Sigma$ and equations (1.6-1.8) are the boundary conditions for the pressure. Notice that conditions $(1.3-1.4)$ can be interpreted as a conservation law for the flux:

$$
\vec{F}=\left(h_{r}+u_{3}\right)^{3} \nabla p-12 \nu_{f}\left(h_{r}+u_{3}\right) \theta(s, 0)
$$

so that (1.5) states the condition $\vec{F} \cdot \vec{n}=0$ on the free boundary.

The previous set of equations is completed with the Koiter model which is posed in its variational form in next section and relates the fluid pressure and the thin bearing displacement.

\subsection{The variational formulation}

In this section we pose the weak formulation for an elastohydrodynamic Reynolds-Koiter problem in order to obtain the existence of a weak solution. First, in view of the equations (1.3-1.8) and taking into account the variational formulation for the Koiter model for shells, let us introduce the usual function spaces and sets:

$$
\begin{aligned}
V_{a} & =\left\{\varphi \in H^{1}(\Omega) / \varphi\left(x_{1}, L / 2\right)=p_{a}, \varphi\left(x_{1}, 0\right)=0, \varphi\left(0, x_{2}\right)=\varphi\left(2 \pi R_{m}, x_{2}\right) \quad \text { if } 0<x_{2}<L / 2\right\} \\
V_{0} & =\left\{\varphi \in H^{1}(\Omega) / \varphi\left(x_{1}, L / 2\right)=\varphi\left(x_{1}, 0\right)=0, \varphi\left(0, x_{2}\right)=\varphi\left(2 \pi R_{m}, x_{2}\right) \quad \text { if } 0<x_{2}<L / 2\right\} \\
V_{t} & =\left\{v_{t}=v^{\alpha} \overrightarrow{a_{\alpha}} / v^{\alpha} \in H^{1}(\widetilde{\Omega}), v^{\alpha}=0 \text { on } \Gamma_{b} \cup \Gamma_{c}\right\} \\
V_{3}^{*} & =\left\{v \in H^{2}(\widetilde{\Omega}) / v=v_{, \alpha}=0 \text { on } \Gamma_{b} \cup \Gamma_{c}\right\} \\
W(\widetilde{\Omega}) & =V_{t} \times V_{3}^{*}
\end{aligned}
$$

where Greek subscripts belong to $\{1,2\}$ and $\left\{\overrightarrow{a_{1}}, \overrightarrow{a_{2}}\right\}$ is a basis of the tangent plane to the middle surface $\omega$.

Let $\vec{u}=\left(u_{t}, u_{3}\right)$ be the displacement field of the bearing middle surface points, its components being referred to the local orthogonal basis $\left\{\overrightarrow{a_{1}}, \overrightarrow{a_{2}}, \overrightarrow{a_{3}}\right\}$. A weak formulation for the coupled Reynolds-Koiter model can be 
written as follows:

Find $(p, \theta, \vec{u}) \in V_{a} \times L^{\infty}(\Omega) \times W(\widetilde{\Omega})$ such that:

$$
(\mathcal{P}) \begin{cases}\int_{\Omega}\left(h_{r}+u_{3}\right)^{3} \nabla p \nabla \varphi=12 \nu_{f} s \int_{\Omega}\left(h_{r}+u_{3}\right) \theta \frac{\partial \varphi}{\partial x_{1}}, & \forall \varphi \in V_{0} \\ \theta \in \mathcal{H}(p) & \text { in } \Omega \\ \int_{\widetilde{\Omega}} R^{M} \gamma_{t}(\vec{u}) \gamma_{t}(\vec{v}) \sqrt{g}+\int_{\tilde{\Omega}} R^{F} \rho(\vec{u}) \rho(\vec{v}) \sqrt{g}=\int_{\tilde{\Omega}} \widetilde{p} v_{3} \sqrt{g}, & \forall \vec{v} \in W(\widetilde{\Omega})\end{cases}
$$

where $\mathcal{H}$ represents the multivalued Heaviside operator:

$$
\mathcal{H}(s)= \begin{cases}0, & \text { if } s<0 \\ {[0,1],} & \text { if } s=0 \\ 1, & \text { if } s>0\end{cases}
$$

$\widetilde{p}$ is the symmetric extension of $p$ to $\widetilde{\Omega}$ (see prolongation operator in Sect. 2.1) and $g$ is the determinant of the metric tensor; notice that $g=1$ for the usual parametrization. The components of the plane deformation and change of curvature tensors in Koiter model are given by:

$$
\begin{aligned}
& \gamma_{\alpha \beta}(\vec{u})=\frac{1}{2}\left(u_{\alpha \mid \beta}+u_{\beta \mid \alpha}\right)-b_{\alpha \beta} u_{3} \\
& \rho_{\alpha \beta}(\vec{u})=\frac{1}{2}\left(\eta_{\alpha \mid \beta}+\eta_{\beta \mid \alpha}\right)+\frac{1}{2}\left(b_{\beta}^{\lambda} u_{\alpha \mid \lambda}+b_{\alpha}^{\lambda} u_{\beta \mid \lambda}\right)-b_{\alpha}^{\lambda} b_{\beta \lambda} u_{3},
\end{aligned}
$$

the covariant derivative is

$$
u_{\alpha \mid \beta}=u_{\alpha, \beta}-\Gamma_{\alpha \beta}^{\lambda} u_{\lambda}
$$

(the same for $\eta_{\alpha \mid \beta}$ ), and the normal rotation vector $\vec{\eta}$ is given by Kirchhoff-Love relation:

$$
\eta_{\alpha}=-u_{3, \alpha}-b_{\alpha}^{\lambda} u_{\lambda}
$$

In previous equations, $b_{\alpha \beta}$ are the components of the curvature tensor, while $\Gamma_{\alpha \beta}^{\lambda}$ are Christoffel symbols. Finally, $R^{M}$ and $R^{F}$ are the membrane and the flexion rigidity tensors [11].

Notice that the coupled feature arises from both the presence of the unknown $\widetilde{p}$ in the second member of the Koiter model and the contribution of the unknown $u_{3}$ to the Reynolds equation coefficients.

When obtaining the existence of solution for the weak formulation in $(\mathcal{P})$, several difficulties arise: the coupled aspect of the problem, the multivalued nonlinearity associated to the Reynolds model and the presence of different sets, namely $\Omega$ and $\widetilde{\Omega}$, in the involved variational identities.

Thus, as in other equations involving the nonlinear multivalued Heaviside operator, we will apply a regularization procedure to the multivalued operator which leads to a regularized coupled problem. Next, the elastic part (Koiter model) and the hydrodynamic part (Elrod-Adams model for Reynolds equation) of the problem can be uncoupled to obtain the solution as a fixed point. This approach requires the mathematical analysis of both problems in order to obtain the existence, the uniqueness and the estimates for their respective solutions. An additional feature involved in the particular Reynolds-Koiter model here proposed is the formulation of the elastic and hydrodynamic subproblems over different sets. This aspect is solved in next sections by using a symmetric prolongation result for the pressure $p$ and the restriction operator for the normal displacement $u_{3}$. 


\subsection{Regularized problem}

As in other previously treated elastohydrodynamic journal bearing problems (see $[6,14]$ ) we propose the following regularization procedure to cope with the difficulty associated to the nonlinear multivalued relation between pressure and saturation unknowns. Thus, for $\varepsilon>0$ let us consider the $\varepsilon$-dependent Lipschitz function:

$$
\mathcal{H}_{\varepsilon}(s)= \begin{cases}0, & \text { if } s<0 \\ s / \varepsilon, & \text { if } 0 \leq s \leq \varepsilon \\ 1, & \text { if } s>\varepsilon .\end{cases}
$$

So, the regularized problem is posed as follows:

Find $\left(p^{\varepsilon}, \vec{u}^{\varepsilon}\right) \in V_{a} \times W(\widetilde{\Omega})$ such that:

$$
\begin{cases}\int_{\Omega}\left(h_{r}+u_{3}^{\varepsilon}\right)^{3} \nabla p^{\varepsilon} \nabla \varphi=12 \nu_{f} s \int_{\Omega}\left(h_{r}+u_{3}^{\varepsilon}\right) \mathcal{H}_{\varepsilon}\left(p^{\varepsilon}\right) \frac{\partial \varphi}{\partial x_{1}}, & \forall \varphi \in V_{0} \\ \int_{\widetilde{\Omega}} R^{M} \gamma_{t}\left(\vec{u}^{\varepsilon}\right) \gamma_{t}(\vec{v})+\int_{\widetilde{\Omega}} R^{F} \rho\left(\vec{u}^{\varepsilon}\right) \rho(\vec{v})=\int_{\widetilde{\Omega}} \widetilde{p}^{\varepsilon} v_{3}, & \forall \vec{v} \in W(\widetilde{\Omega}) .\end{cases}
$$

Notice that the $\varepsilon$-dependent regularized problem $\left(\mathcal{P}_{\varepsilon}\right)$ remains to be a coupled problem with each variational identity posed on a different domain. Section 2 is devoted to the mathematical analysis of problem $\left(\mathcal{P}_{\varepsilon}\right)$ in order to obtain an existence result and the appropriate estimates for the solution. This will allow to pass to the limit in the regularization parameter and state the existence of solution for the problem $(\mathcal{P})$ in Section 3.

\section{Existence of SOLUTION FOR THE REGUlaRIZED PROBLEM}

\subsection{Fixed point operator}

In order to prove the existence of solution for the regularized problem $\left(\mathcal{P}_{\varepsilon}\right)$, we use in this section a fixed point technique. Thus, a solution is obtained as a fixed point for an appropriate compact operator. Although this problem depends on $\varepsilon$, in most of this section we drop the $\varepsilon$ index for simplicity.

First, for a given $R$ to be determined, we define the set

$$
B_{R}=\left\{\varphi \in L^{2}(\Omega) / 0 \leq \varphi \leq R\right\}
$$

and we introduce the operator $T: B_{R} \longrightarrow T\left(B_{R}\right)$ as the composition operator:

$$
T=T_{4} \circ T_{3} \circ T_{2} \circ T_{1}
$$

where the involved operators are:

(a) Prolongation operator $T_{1}$ :

$$
\begin{aligned}
T_{1}: B_{R} & \longrightarrow L^{2}(\widetilde{\Omega}) \\
p & \longrightarrow T_{1}(p)=\widetilde{p},
\end{aligned}
$$

with $\widetilde{p}$ defined by

$$
\widetilde{p}\left(x_{1}, x_{2}\right)= \begin{cases}p\left(x_{1}, x_{2}\right) & \text { if } x_{2} \in(0, L / 2) \\ p\left(x_{1}, L-x_{2}\right) & \text { if } x_{2} \in(L / 2, L)\end{cases}
$$

This operator extends by symmetry a function $p$ to $\widetilde{\Omega}$. 
(b) Koiter operator $T_{2}$ :

$$
\begin{aligned}
T_{2}: T_{1}\left(B_{R}\right) & \longrightarrow W(\widetilde{\Omega}) \\
\widetilde{p} & \longrightarrow T_{2}(\widetilde{p})=\vec{u}
\end{aligned}
$$

where $\vec{u}$ is the unique solution of the Koiter model:

Find $\vec{u} \in W(\widetilde{\Omega})$ such that:

$$
\int_{\tilde{\Omega}} R^{M} \gamma_{t}(\vec{u}) \gamma_{t}(\vec{v})+\int_{\tilde{\Omega}} R^{F} \rho(\vec{u}) \rho(\vec{v})=\int_{\tilde{\Omega}} \widetilde{p} v_{3} \quad, \quad \forall \vec{v} \in W(\widetilde{\Omega}) .
$$

Notice that the second member represents the external force, due to pressure $\widetilde{p}$. More precisely, as $\overrightarrow{a_{3}}$ denotes the normal vector to the shell middle surface, we have:

$$
\left(\widetilde{p} \overrightarrow{a_{3}}\right) \cdot \vec{v}=\widetilde{p}\left(\overrightarrow{a_{3}} \cdot \vec{v}\right)=\widetilde{p} v_{3}
$$

(c) Restriction to $\Omega$ of the normal displacement operator $T_{3}$ :

$$
\begin{aligned}
& T_{3}:\left(T_{2} \circ T_{1}\right)\left(B_{R}\right) \longrightarrow H^{2}(\Omega) \\
& \vec{u} \quad \longrightarrow T_{3}(\vec{u})=u_{3} \text {. }
\end{aligned}
$$

(d) Lubrication operator $T_{4}$ :

$$
\begin{aligned}
& T_{4}:\left(T_{3} \circ T_{2} \circ T_{1}\right)\left(B_{R}\right) \longrightarrow \quad L^{2}(\Omega) \\
& u_{3} \quad \longrightarrow T_{4}\left(u_{3}\right)=q
\end{aligned}
$$

where, for a given $p \in B_{R}$, the function $q$ is the unique solution of the linear problem:

Find $q \in V_{a}$ such that:

$$
\int_{\Omega}\left(h_{r}+u_{3}\right)^{3} \nabla q \nabla \varphi=C \int_{\Omega}\left(h_{r}+u_{3}\right) \mathcal{H}_{\varepsilon}(p) \frac{\partial \varphi}{\partial x_{1}}, \quad \forall \varphi \in V_{0}
$$

with $C=12 \nu_{f} s$ a known constant.

The idea is to establish an appropriate hypothesis on the data of the regularized problem, so that a fixed point for the operator $T$ exists for a convenient choice of $R$. For this, an $L^{\infty}$ estimate for the solution of (2.2) is required. In view of the definition of the previous operators, this estimate depends on the obtained estimates for the solutions of the succesive problems which are posed in terms of $p \in B_{R}$. Thus, we will proceed to the study of the different operators to get the corresponding estimates at each step.

\subsection{Operator splitting}

In this section we analize the properties of the operators involved in the factorization of $T$. As a result we obtain that under certain conditions $T$ is a compact operator from $B_{R}$ into $B_{R}$.

\subsubsection{Prolongation operator}

The operator $T_{1}$ extends to $L^{2}(\widetilde{\Omega})$ any function belonging to $L^{2}(\Omega)$, by simmetry with respect to $\Gamma_{a}$. From the classical prolongation result (see Lem. IX.2 in [9]) the inequality

$$
\left\|T_{1}(p)\right\|_{L^{2}(\tilde{\Omega})} \leq K_{1}\|p\|_{L^{2}(\Omega)}
$$

follows. So, the following lemma states an $L^{2}$ estimate for $T_{1}(p)$ when $p \in B_{R}$. 
Lemma 2.1. If $p \in B_{R}$ then $\left\|T_{1}(p)\right\|_{L^{2}(\widetilde{\Omega})} \leq K_{2} R$, where $K_{2}=|\Omega|^{1 / 2} K_{1}$.

\subsubsection{Koiter operator}

For a given pressure $\widetilde{p}$ defined in $\widetilde{\Omega}$, the operator $T_{2}$ provides the bearing displacement field $\vec{u}$. In order to study operator $T_{2}$, following the classical notation for Koiter model (see [11], for example) we introduce the bilinear form

$$
\mathcal{B}(\vec{u}, \vec{v})=\int_{\tilde{\Omega}} R^{M} \gamma_{t}(\vec{u}) \gamma_{t}(\vec{v})+\int_{\tilde{\Omega}} R^{F} \rho(\vec{u}) \rho(\vec{v}), \quad \vec{u}, \vec{v} \in W(\widetilde{\Omega})
$$

and the linear form

$$
l(\vec{v})=\int_{\widetilde{\Omega}} \widetilde{p} v_{3}, \quad \vec{v} \in W(\widetilde{\Omega}) .
$$

So, as $\mathcal{B}$ is a coercive bilinear form $[7,8]$, there exists a positive constant $\beta$ such that

$$
\mathcal{B}(\vec{u}, \vec{u}) \geq \beta\|\vec{u}\|_{W(\tilde{\Omega})}^{2} .
$$

By using the previous inequality we can state the estimate for $\vec{u}$ in the usual norm of $W(\widetilde{\Omega})$ when $p \in B_{R}$. Notice that for simplicity we drop a rigorous notation including the dependence of $\vec{u}$ on $p$.

Lemma 2.2. If $p \in B_{R}$, then $\|\vec{u}\|_{W(\tilde{\Omega})} \leq \beta^{-1} K_{2} R$.

Proof. It is straightforward from Hölder inequality.

\subsubsection{Restriction operator}

For a given displacement field $\vec{u}$ in $\widetilde{\Omega}$, the operator $T_{3}$ assigns the restriction to $\Omega$ of the third component, $u_{3}$, of this displacement vector. So, the inequality

$$
\left\|T_{3}(\vec{u})\right\|_{H^{2}(\Omega)} \leq\|\vec{u}\|_{W(\tilde{\Omega})}
$$

holds and we can conclude that $T_{3}$ is continuous. Moreover we have the following result.

Lemma 2.3. If $p \in B_{R}$ then the $L^{\infty}$-estimate

$$
\left\|u_{3}\right\|_{L^{\infty}(\Omega)} \leq K \beta^{-1} R
$$

holds with $K=K(\Omega)$.

Proof. It is straightforward for $K=K_{2} K_{3}$, being $K_{3}$ the constant associated to the continuous inclusion $i: H^{2}(\Omega) \rightarrow L^{\infty}(\Omega)$ (see [16], for the inclusion result).

Again the dependence $u_{3}=u_{3}(p)$ is not explicit in the notation. 


\subsubsection{Lubrication operator}

For $p \in B_{R}$, the operator $T_{4}$ associates to a given normal displacement, $u_{3}$, the function $q \in L^{2}(\Omega)$ which is the solution of the problem:

Find $q \in V_{a}$ such that:

$$
\int_{\Omega}\left(h_{r}+u_{3}\right)^{3} \nabla q \nabla \varphi=C \int_{\Omega}\left(h_{r}+u_{3}\right) \mathcal{H}_{\varepsilon}(p) \frac{\partial \varphi}{\partial x_{1}}, \quad \forall \varphi \in V_{0}
$$

Proposition 2.4. For $p \in B_{R}$ such that $h_{r}-K \beta^{-1} R>0$, the problem (2.5) has a unique nonnegative solution. Moreover, this solution verifies the estimates:

$$
\begin{aligned}
\|\nabla q\|_{L^{2}(\Omega)} & \leq\left[\frac{C+2 p_{a} L^{-1}\left(\overline{h_{r}}+K \beta^{-1} R\right)^{2}}{\left(\underline{h_{r}}-K \beta^{-1} R\right)^{2}}\right]|\Omega|^{1 / 2} \\
\|q\|_{L^{\infty}(\Omega)} & \leq p_{a}+\frac{C C_{r^{*}}|\Omega|^{\frac{1}{2}-\frac{1}{r^{*}}} 2^{\frac{r^{*}}{r^{*}-2}}}{\left.\underline{h_{r}}-K \beta^{-1} R\right)^{2}}
\end{aligned}
$$

where $C_{r^{*}}$ is the constant associated to the continuous inclusion $V_{0} \longrightarrow L^{r^{*}}(\Omega)$ for $r^{*}>2$.

Proof. First, we point out that the problem defined by equation (6) is posed for the prescribed functions $p$ and $u_{3}$. So, it can be analyzed as the regularized problem for the hydrodynamic Elrod-Adams model associated to the modified gap function $h_{r}+u_{3}$. Thus, the existence and uniqueness of a nonnegative solution can be obtained by using the same techniques than those in [1] for an hydrodynamic regularized problem with circumferential supply. Next, in order to obtain the $H^{1}(\Omega)$ estimate (2.6), let us consider the test function $\varphi=q-2 p_{a} L^{-1} x_{2}$ in (2.5). Thus, we have

$$
\int_{\Omega}\left(h_{r}+u_{3}\right)^{3}|\nabla q|^{2}=C \int_{\Omega}\left(h_{r}+u_{3}\right) \mathcal{H}_{\varepsilon}(p) \frac{\partial q}{\partial x_{1}}+2 p_{a} L^{-1} \int_{\Omega}\left(h_{r}+u_{3}\right)^{3} \frac{\partial q}{\partial x_{2}}
$$

and, therefore

$$
\begin{aligned}
\left.\underline{\left(h_{r}\right.}-K \beta^{-1} R\right) \int_{\Omega}\left(h_{r}+u_{3}\right)^{2}|\nabla q|^{2} & \leq \int_{\Omega}\left(h_{r}+u_{3}\right)^{3}|\nabla q|^{2} \\
& =C \int_{\Omega}\left(h_{r}+u_{3}\right) \mathcal{H}_{\varepsilon}(p)\left|\frac{\partial q}{\partial x_{1}}\right|+2 p_{a} L^{-1} \int_{\Omega}\left(h_{r}+u_{3}\right)^{3}\left|\frac{\partial q}{\partial x_{2}}\right| \\
& \leq C \int_{\Omega}\left|h_{r}+u_{3}\right||\nabla q|+2 p_{a} L^{-1}\left(\overline{h_{r}}+K \beta^{-1} R\right)^{2} \int_{\Omega}\left|h_{r}+u_{3}\right||\nabla q| \\
& \leq\left[C+2 p_{a} L^{-1}\left(\overline{h_{r}}+K \beta^{-1} R\right)^{2}\right]|\Omega|^{1 / 2}\left[\int_{\Omega}\left|h_{r}+u_{3}\right|^{2}|\nabla q|^{2}\right]^{1 / 2}
\end{aligned}
$$

where the last inequality follows from Holder's one. So,

$$
\left[\int_{\Omega}\left|h_{r}+u_{3}\right|^{2}|\nabla q|^{2}\right]^{1 / 2} \leq \frac{\left[C+2 p_{a} L^{-1}\left(\overline{h_{r}}+K \beta^{-1} R\right)^{2}\right]|\Omega|^{1 / 2}}{\left(\underline{h_{r}}-K \beta^{-1} R\right)}
$$

and the estimate (2.6) is easily concluded.

Next, in order to obtain the $L^{\infty}(\Omega)$ estimate for $q$, we use the technique previously developed in [14] for the Reynolds-plate coupled model, which is based on $L^{\infty}$ estimates for elliptic variational equations, (see [18], for 
example). Thus, for $k>p_{a}>0$, we define the set $\Omega_{k}=\left\{\left(x_{1}, x_{2}\right) \in \Omega / q\left(x_{1}, x_{2}\right)>k\right\}$, and we take $\xi_{k}=(q-k)^{+}$ as test function in (2.5). So,

$$
\begin{aligned}
\left.\underline{\left(h_{r}\right.}-K \beta^{-1} R\right) \int_{\Omega}\left(h_{r}+u_{3}\right)^{2}\left|\nabla \xi_{k}\right|^{2} & \leq C \int_{\Omega_{k}}\left(h_{r}+u_{3}\right)\left|\frac{\partial \xi_{k}}{\partial x_{1}}\right| \\
& \leq C\left|\Omega_{k}\right|^{1 / 2}\left[\int_{\Omega_{k}}\left(h_{r}+u_{3}\right)^{2}\left|\frac{\partial \xi_{k}}{\partial x_{1}}\right|^{2}\right]^{1 / 2} \\
& \leq C\left|\Omega_{k}\right|^{1 / 2}\left[\int_{\Omega}\left(h_{r}+u_{3}\right)^{2}\left|\nabla \xi_{k}\right|^{2}\right]^{1 / 2}
\end{aligned}
$$

and, finally, we have:

$$
\left\|\nabla \xi_{k}\right\|_{L^{2}(\Omega)} \leq C \frac{\left|\Omega_{k}\right|^{1 / 2}}{\left(\underline{h_{r}}-K \beta^{-1} R\right)^{2}} .
$$

Next, for $k_{1}>k_{2}>p_{a}>0$ we have $\Omega_{k_{1}} \subset \Omega_{k_{2}}$ and then

$$
\left(k_{1}-k_{2}\right)^{r^{*}}\left|\Omega_{k_{1}}\right|=\int_{\Omega_{k_{1}}}\left(k_{1}-k_{2}\right)^{r^{*}} \leq \int_{\Omega_{k_{1}}}\left(q-k_{2}\right)^{r^{*}} \leq \int_{\Omega}\left|\xi_{k_{2}}\right|^{r^{*}} .
$$

Now, by choosing $r^{*}>2$, from the continuous inclusion $i_{r^{*}}: V_{0} \hookrightarrow L^{r^{*}}(\Omega)$, we have

$$
\left(k_{1}-k_{2}\right)^{r^{*}}\left|\Omega_{k_{1}}\right| \leq\left\|\xi_{k_{2}}\right\|_{L^{r^{*}}(\Omega)}^{r^{*}} \leq C_{r^{*}}^{r^{*}}\left\|\nabla \xi_{k_{2}}\right\|_{L^{2}(\Omega)}^{r^{*}} \leq\left[C_{r^{*}} \frac{C\left|\Omega_{k_{2}}\right|^{1 / 2}}{\left.\underline{\left(h_{r}\right.}-K \beta^{-1} R\right)^{2}}\right]^{r^{*}}
$$

where $C_{r^{*}}$ is the Sobolev constant for the inclusion. So, we can state

$$
\left|\Omega_{k_{1}}\right| \leq\left[C_{r^{*}} \frac{C}{\left(\underline{h_{r}}-K \beta^{-1} R\right)^{2}}\right]^{r^{*}} \frac{1}{\left(k_{1}-k_{2}\right)^{r^{*}}}\left|\Omega_{k_{2}}\right|^{r^{*} / 2} .
$$

Now, applying Lemma B.1 of [18] we get the estimate (2.7).

\subsection{Existence of the fixed point}

In order to apply the Schauder fixed point theorem for operator $T$, we establish the following technical lemma.

Lemma 2.5. If the data of the coupled problem verify the hypotheses:

$$
\begin{aligned}
& p_{a}<K^{-1} \beta \underline{h_{r}}, \\
& \frac{12 \underline{h_{r}} p_{a} K \beta^{-1}\left(\underline{h_{r}}-p_{a} K \beta^{-1}\right)+27 C_{1}(\Omega) K \beta^{-1}}{4\left[{\underline{h_{r}}}^{3}-\left(p_{a} K \beta^{-1}\right)^{3}\right]}<1,
\end{aligned}
$$

then there exists:

$$
R_{1}=\frac{\beta}{3 K}\left(2 p_{a} K \beta^{-1}+\underline{h_{r}}\right) \in\left(0, K^{-1} \underline{\beta h_{r}}\right)
$$

such that $P\left(R_{1}\right)>0$ for the polynomial

$$
\left.P(R)=K^{2} \beta^{-2} R^{3}-\left(p_{a} K^{2} \beta^{-2}+2 K \beta^{-1} \underline{h_{r}}\right) R^{2}+{\underline{h_{r}}}^{2}+2 K \beta^{-1} p_{a} \underline{h_{r}}\right) R-\left(p_{a}{\underline{h_{r}}}^{2}+C_{1}(\Omega)\right),
$$


where $C_{1}(\Omega)=C C_{r^{*}}|\Omega|^{\frac{1}{2}-\frac{1}{r^{*}}} 2^{\frac{r^{*}}{r^{*}-2}}$ for clearness.

Proof. Notice that, from the hypotheses $(\mathrm{H} 1)$ and $(\mathrm{H} 2)$, the polynomial $P^{\prime}$ has two positive real roots. Let $R_{1}$ be the smallest root, which is given by:

$$
\begin{aligned}
R_{1} & =\frac{\beta^{2}}{3 K^{2}}\left[\left(p_{a} K^{2} \beta^{-2}+2 K \beta^{-1} \underline{h_{r}}\right)-\sqrt{\left(p_{a} K^{2} \beta^{-2}+2 K \beta^{-1} \underline{h_{r}}\right)^{2}-3 K^{2} \beta^{-2}\left(\underline{h_{r}^{2}}+2 p_{a} K \beta^{-1} \underline{h_{r}}\right)}\right] \\
& =\frac{\beta^{2}}{3 K^{2}}\left[\left(p_{a} K^{2} \beta^{-2}+2 K \beta^{-1} \underline{h_{r}}\right)-\sqrt{\left(K \beta^{-1} \underline{h_{r}}-p_{a} K^{2} \beta^{-2}\right)^{2}}\right] .
\end{aligned}
$$

Moreover, from (H1), we have

$$
R_{1}=\frac{\beta}{3 K}\left(2 p_{a} K \beta^{-1}+\underline{h_{r}}\right)<\underline{h_{r}} K^{-1} \beta
$$

and, from easy computations, we obtain that $P\left(R_{1}\right)>0$.

Next, under some hypotheses on the data of the coupled problem, we state the existence of solution for the regularized problem.

Theorem 2.6. If the data of the coupled problem $(\mathcal{P})$ verify the hypotheses:

$$
\begin{aligned}
& p_{a}<K^{-1} \beta \underline{h_{r}}, \\
& \frac{12 \underline{h_{r}} p_{a} K \beta^{-1}\left(\underline{h_{r}}-p_{a} K \beta^{-1}\right)+27 C_{1}(\Omega) K \beta^{-1}}{4\left[\underline{h r}^{3}-\left(p_{a} K \beta^{-1}\right)^{3}\right]}<1,
\end{aligned}
$$

with $K=K(\Omega)$ then there exists a fixed point, $p^{\varepsilon}$, for the operator $T$, which is the solution of the regularized problem $\left(\mathcal{P}_{\varepsilon}\right)$. Moreover, we have the estimates:

$$
\begin{array}{r}
\left\|\nabla p^{\varepsilon}\right\|_{L^{2}(\Omega)} \leq \frac{9 C+2 p_{a} L^{-1}\left(3 \overline{h_{r}}+\underline{h_{r}}+2 K \beta^{-1} p_{a}\right)^{2}}{\left.4 \underline{h_{r}}-K \beta^{-1} p_{a}\right)^{2}}|\Omega|^{1 / 2}=A_{1} \\
\left\|p^{\varepsilon}\right\|_{L^{\infty}(\Omega)} \leq p_{a}+\frac{9 C C_{r^{*}}|\Omega|^{\frac{1}{2}-\frac{1}{r^{*}}} 2^{\frac{4-r^{*}}{r^{*}-2}}}{\left(\underline{h_{r}}-K \beta^{-1} p_{a}\right)^{2}}=A_{2}
\end{array}
$$

where $A_{1}$ and $A_{2}$ are constants which do not depend on $\varepsilon$.

Proof. First, from hypothesis (H1), Proposition 2.4 implies that the operator $T$ is well defined. Moreover, from the hypotheses (H1) and (H2), the previous lemma states that

$$
P\left(R_{1}\right)=K^{2} \beta^{-2} R_{1}^{3}-\left(p_{a} K^{2} \beta^{-2}+2 K \beta^{-1} \underline{h_{r}}\right) R_{1}^{2}+\left({\underline{h_{r}}}^{2}+2 K \beta^{-1} p_{a} \underline{h_{r}}\right) R_{1}-\left(p_{a}{\underline{h_{r}}}^{2}+C_{1}(\Omega)\right)>0
$$

which is equivalent to the inequality

$$
p_{a}+\frac{C_{1}(\Omega)}{\left(\underline{h_{r}}-K \beta^{-1} R_{1}\right)^{2}}<R_{1}
$$

so that $T\left(B_{R_{1}}\right) \subset B_{R_{1}}$. Indeed, $T$ is a continuous and compact operator (notice that, for example, $T_{4}$ is compact). From Schauder fixed point theorem there exists $p \in B_{R_{1}}$ such that $p=T(p)$. So, the couple $\left(p^{\varepsilon}=p, \vec{u}^{\varepsilon}=\left(T_{2} \circ T_{1}\right)(p)\right)$ provides a solution of the regularized problem $\left(\mathcal{P}_{\varepsilon}\right)$.

Finally, from (2.6) and (2.7) in Proposition 2.4 for $R=R_{1}$, we easily get the estimates (2.9) and (2.10). 


\section{Existence of SOlution FOR THE COUPLED PROBlem}

In this section, the main result of the paper is stated. Thus, under the hypotheses (H1) and (H2) on the data, the existence of a solution for problem $(\mathcal{P})$ is concluded as a limit of solutions for the problems $\left(\mathcal{P}_{\varepsilon}\right)$. Notice that the obtained solution is also characterized as the limit of a sequence of fixed points.

Theorem 3.1. If the data of the coupled problem $(\mathcal{P})$ verify the hypotheses (H1) and (H2), then there exists a solution $(p, \theta, \vec{u})$ of problem $(\mathcal{P})$. Moreover, the estimates:

$$
\begin{aligned}
\|\nabla p\|_{L^{2}(\Omega)} & \leq \frac{9 C+2 p_{a} L^{-1}\left(3 \overline{h_{r}}+\underline{h_{r}}+2 K \beta^{-1} p_{a}\right)^{2}}{4\left(\underline{h_{r}}-K \beta^{-1} p_{a}\right)^{2}}|\Omega|^{1 / 2} \\
\|p\|_{L^{\infty}(\Omega)} & \leq p_{a}+\frac{9 C C_{r^{*}}|\Omega|^{\frac{1}{2}-\frac{1}{r^{*}}} 2^{\frac{4-r^{*}}{r^{*}-2}}}{\left(\underline{h_{r}}-K \beta^{-1} p_{a}\right)^{2}}
\end{aligned}
$$

hold.

Proof. First, notice that the operator $T$ actually depends on $\varepsilon$, so that we have a sequence of fixed points $\left\{p^{\varepsilon}\right\}_{\varepsilon}$. For each $\varepsilon>0$ let $\vec{u}^{\varepsilon}=T_{2}\left(T_{1}\left(p^{\varepsilon}\right)\right)$.

So, from estimates (2.9) and (2.10) and Lemma 2.2 we can deduce the existence of the subsequences of $\left\{p^{\varepsilon}\right\}_{\varepsilon}$ and $\left\{\vec{u}^{\varepsilon}\right\}_{\varepsilon}$, still noted as their respective sequences, such that:

$$
\begin{array}{rll}
\exists p \in V_{a} \cap L^{\infty}(\Omega), & p^{\varepsilon} \longrightarrow p & \text { in } H^{1}(\Omega) \text { weakly and } L^{\infty}(\Omega) \text { weakly - *, } \\
\exists \vec{u} \in W(\widetilde{\Omega}), & \vec{u}^{\varepsilon} \longrightarrow \vec{u} & \text { in } W(\widetilde{\Omega}) \text { weakly and } L^{\infty}(\widetilde{\Omega}) \text { weakly - *. }
\end{array}
$$

Indeed, the last convergence implies that $u_{3}^{\varepsilon} \longrightarrow u_{3}$ in $H^{2}(\Omega)$ weakly.

On the other hand, as the trivial bound $0 \leq \mathcal{H}_{\varepsilon}\left(p^{\varepsilon}\right) \leq 1$ holds, then

$$
\exists \theta \in L^{\infty}(\Omega), \quad \mathcal{H}_{\varepsilon}\left(p^{\varepsilon}\right) \longrightarrow \theta \quad \text { in } L^{\infty}(\Omega) \text { weakly -*. }
$$

The set of the above convergences allows us to pass to the limit in the parameter $\varepsilon$ in problem $\left(\mathcal{P}_{\varepsilon}\right)$. More precisely, we have

$$
\begin{aligned}
\int_{\Omega}\left(h_{r}+u_{3}^{\varepsilon}\right)^{3} \nabla p^{\varepsilon} \nabla \varphi & \longrightarrow \int_{\Omega}\left(h_{r}+u_{3}\right)^{3} \nabla p \nabla \varphi \\
\int_{\Omega}\left(h_{r}+u_{3}^{\varepsilon}\right) \mathcal{H}_{\varepsilon}\left(p^{\varepsilon}\right) \frac{\partial \varphi}{\partial x_{1}} & \longrightarrow \int_{\Omega}\left(h_{r}+u_{3}\right) \theta \frac{\partial \varphi}{\partial x_{1}} \\
\int_{\tilde{\Omega}} R^{M} \gamma_{t}\left(\vec{u}^{\varepsilon}\right) \gamma_{t}(\vec{v})+\int_{\tilde{\Omega}} R^{F} \rho\left(\vec{u}^{\varepsilon}\right) \rho(\vec{v}) & \longrightarrow \int_{\tilde{\Omega}} R^{M} \gamma_{t}(\vec{u}) \gamma_{t}(\vec{v})+\int_{\tilde{\Omega}} R^{F} \rho(\vec{u}) \rho(\vec{v}) \\
\int_{\tilde{\Omega}} \widetilde{p}^{\varepsilon} v_{3} & \longrightarrow \int_{\tilde{\Omega}} \widetilde{p} v_{3}
\end{aligned}
$$

where (3.3) follows from the convergences $u_{3}^{\varepsilon} \rightarrow u_{3}$ in $H^{2}(\Omega)$ weakly and $p^{\varepsilon} \rightarrow p$ in $H^{1}(\Omega)$ weakly, (3.4) from the limits $u_{3}^{\varepsilon} \rightarrow u_{3}$ weakly and $\mathcal{H}_{\varepsilon}\left(p^{\varepsilon}\right) \rightarrow \theta$ in $L^{\infty}$ weakly, (3.5) follows from the convergence $\vec{u}^{\varepsilon} \rightarrow \vec{u}$ in $H^{2}(\widetilde{\Omega})$ weakly, and (3.6) is a consequence of the convergence $\widetilde{p}^{\varepsilon} \rightarrow \widetilde{p}$ in $H^{1}(\widetilde{\Omega})$ weakly.

Moreover, if we consider that

$$
0 \leq \int_{\Omega} p^{\varepsilon}\left(1-\mathcal{H}_{\varepsilon}\left(p^{\varepsilon}\right)\right) \mathrm{d} \Omega=\int_{\left|p^{\varepsilon}<\varepsilon\right|} p^{\varepsilon}\left(1-\mathcal{H}_{\varepsilon}\left(p^{\varepsilon}\right)\right) \mathrm{d} \Omega \leq \int_{\left|p^{\varepsilon}<\varepsilon\right|} \varepsilon \mathrm{d} \Omega \leq \varepsilon|\Omega|,
$$

we can state that $\theta \in \mathcal{H}(p)$. So, we have proved that the limit $(p, \theta, \vec{u})$ is a solution of the coupled problem $(\mathcal{P})$. 
Remark 3.2. The hypothesis (H1) imposes a compatibility condition among the supply pressure $\left(p_{a}\right)$, the rigidity of the shell (associated to constant $\beta$ ) and the minimum reference gap $\left(h_{r}\right)$ which prevents from the appearance of regions where $h_{r}+u_{3} \leq 0$. Although, as in other elastohydrodynamic problems, uniqueness remains to be an open question for this stationary model, the possible presence of unstable phenomena associated to a not enough rigid shell is removed by the constraint (H1) on the parameter $\beta$. More precisely, under hypothesis (H1) the parameter $\beta$ cannot tend freely to zero.

\section{Numerical SOLUtion FOR A KoIter MiXed FORMUlation}

Although the main aim of this paper is to establish the existence of solution for problem $(\mathcal{P})$, a numerical method to approximate a solution and some real data simulations are summarized in this section. In a previous paper [2], a slightly different Reynolds-Koiter coupled model has been first proposed to perform some numerical simulation studies in the frame of imposed load problems. More precisely, in [2] the elastic Koiter problem is posed in the domain $\Omega$ so that a symmetry condition for the normal displacement has to be considered at the supply boundary $\Gamma_{a}$ to obtain realistic simulations.

In the present paper, in order to compute a numerical approximation for the coupled problem $(\mathcal{P})$, we propose — as in other related elastohydrodynamic problems - an explicit iterative scheme which essentialy uncouples the hydrodynamic and elastic parts of the coupled problem. In the particular case here treated, the operators $T_{2}$ and $T_{4}$ represent the elastic and hydrodynamic problems, respectively. In our approach, the operators $T_{1}$ and $T_{3}$ are also numerically implemented. Thus, the numerical method reproduces the previously developed theoretical techniques to obtain the existence result. In this way the approximation of the solution is computed as a fixed point for the discretized operators.

First of all, as the unknown $u_{3}$ belongs to $H^{2}(\widetilde{\Omega})$, the discretization of the problem associated to operator $T_{4}$ requires at least the use of second order finite elements. In this paper, and only for numerical purposes, we replace Koiter operator by the one associated to a mixed formulation developed by Destuynder and Salaün [12]. In this alternative mixed formulation the normal displacement lies in $H^{1}(\widetilde{\Omega})$. The main numerical advantage is the possibility of using $P_{1}$ Lagrange finite elements for the approximation of $\vec{u}$, although an additional degree of freedom is needed to approximate the normal rotation vector. For clearness, the mixed formulation we use for Koiter model is summarized in next paragraphs.

\subsection{The mixed formulation for Koiter shell model}

As in [2], in order to simulate the bearing displacements we replace Koiter model defined by operator $T_{4}$ by a mixed formulation developed in $[12,13]$. So, we introduce the functional space

$$
V_{3}=\left\{v \in H^{1}(\widetilde{\Omega}) / v=v_{, \alpha}=0 \text { on } \Gamma_{b} \cup \Gamma_{c}\right\}
$$

for the unknown $u_{3}$. Notice that the regularity of $u_{3}$ is relaxed. Moreover, let us introduce the following functional spaces:

$$
L_{0}^{2}(\widetilde{\Omega})=\left\{\psi \in L^{2}(\widetilde{\Omega}), \int_{\widetilde{\Omega}} \psi=0\right\} \quad W_{t}=\left\{\mu=\mu^{\alpha} \overrightarrow{a_{\alpha}} / \mu^{\alpha} \in H^{1}(\widetilde{\Omega}), \mu^{\alpha}=0 \text { on } \Gamma_{b} \cup \Gamma_{c}\right\}
$$

Let us define the spaces $\mathcal{V}=V_{t} \times V_{3} \times W_{t}$ and $\mathcal{M}=V_{3} \times L_{0}^{2}(\widetilde{\Omega})$. Now, by introducing the notations $X=$ $\left(u_{\alpha}, u_{3}, \eta_{\alpha}\right) \in \mathcal{V}, Y=\left(v_{\alpha}, v_{3}, \zeta_{\alpha}\right) \in \mathcal{V}$ and $\Lambda=\left(\psi_{1}, \psi_{2}\right) \in \mathcal{M}$, the mixed formulation associated to Koiter 
model (2.1) can be written as:

Find $(X, \Lambda) \in \mathcal{V} \times \mathcal{M}$ such that:

$$
\left\{\begin{aligned}
A(X, Y)+B(\Lambda, Y) & =F(Y), & & \forall Y \in \mathcal{V} \\
B(\Xi, X) & =0, & & \forall \Xi \in \mathcal{M}
\end{aligned}\right.
$$

where the involved bilinear and linear forms are the following:

$$
\begin{aligned}
A(X, Y) & =\int_{\tilde{\Omega}} R^{M_{\alpha \beta \lambda \mu}} \gamma_{\alpha \beta}(X) \gamma_{\lambda \mu}(Y)+\int_{\tilde{\Omega}} R^{F_{\alpha \beta \lambda \mu}} \rho_{\alpha \beta}(X) \rho_{\lambda \mu}(Y) \\
B(\Lambda, Y) & =\frac{\epsilon^{2}}{3}\left[\int_{\tilde{\Omega}} g^{\alpha \lambda} \psi_{1, \lambda}\left(\mu_{\alpha}+b_{\alpha}^{\beta} v_{\beta}+v_{3, \alpha}\right)-\int_{\tilde{\Omega}} \psi_{2}\left(\zeta_{2,1}+\left(b_{2}^{\beta} v_{\beta}\right)_{, 1}-\zeta_{1,2}-\left(b_{1}^{\beta} v_{\beta}\right)_{, 2}\right)\right] \\
F(Y) & =\int_{\tilde{\Omega}} \widetilde{p} v_{3} .
\end{aligned}
$$

For a given function $\widetilde{p} \in L^{2}(\widetilde{\Omega})$, the existence and uniqueness of solution for the above formulation is stated in [12].

\subsection{Numerical solution of the coupled problem without imposed load}

The numerically solved coupled model consists of problem $(\mathcal{P})$ where the last (elastic) equation has been replaced by the previously described mixed formulation. So, the numerical algorithm is sketched as follows:

- Step 0: Initialize $p^{0}, \theta^{0}$ and $\vec{u}^{0}=0$.

- Step $n+1$ : For given $p^{n}, \theta^{n}$ and $\vec{u}^{n}$ :

- Hydrodynamic part: Let $h_{t}^{n+1}=h_{r}+T_{3}\left(u_{3}^{n}\right)$.

Compute $\left(p^{n+1}, \theta^{n+1}\right) \in V_{a} \times L^{\infty}(\Omega)$ by solving

$$
\begin{aligned}
& \int_{\Omega}\left(h_{t}^{n}\right)^{3} \nabla p^{n+1} \nabla \varphi=\int_{\Omega} h_{t}^{n} \theta^{n+1} \frac{\partial \varphi}{\partial x_{1}}, \quad \forall \varphi \in V_{0} \\
& \theta^{n+1} \in \mathcal{H}\left(p^{n+1}\right) \quad \text { in } \Omega
\end{aligned}
$$

- Elastic part: Let $\widetilde{p}^{n+1}=T_{1}\left(p^{n+1}\right)$.

Compute $u_{3}^{n+1}$ as part of the solution of the mixed formulation in $\widetilde{\Omega}$ :

$$
\left\{\begin{aligned}
A\left(X^{n+1}, Y\right)+B\left(\Lambda^{n+1}, Y\right) & =F(Y), & & \forall Y \in \mathcal{V} \\
B\left(\Xi, X^{n+1}\right) & =0, & & \forall \Xi \in \mathcal{M}
\end{aligned}\right.
$$

For the numerical solution of the free boundary problem (4.2-4.3) we have combined a characteristics algorithm for the time semidiscretization of nonlinear convection problems (see [5], for its numerical analysis), Lagrange $P_{1}$ finite elements for space discretization and a duality method for maximal monotone operators to approximate the couple $\left(p^{n+1}, \theta^{n+1}\right)$. This approach for the hydrodynamic part has been already succesfully used in previously treated elastohydrodynamic problems $[2,15]$. On the other hand, the mixed formulation allows the use of $P_{1}$ finite elements to approximate $\vec{u}^{n+1}$ and $\Lambda^{n+1}$, although a bubble function has to be chosen in order to perform an appropriate approximation of $\eta^{n+1}$ (see [13], for further details). 

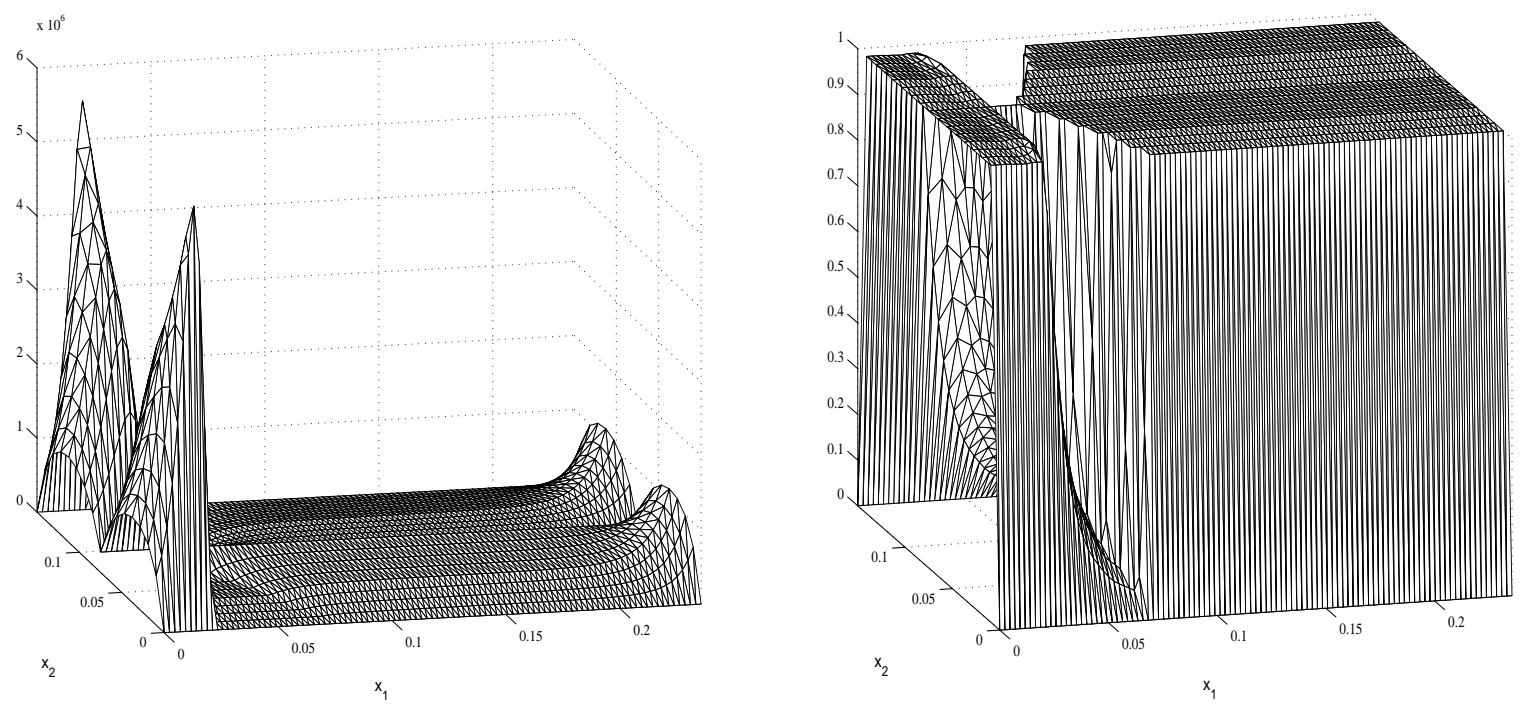

Figure 2. Pressure and concentration without imposed load.

\subsection{Numerical results}

In this section, we present a numerical test that shows the good performance of the previously described algorithm. We simulate a journal-bearing device whose length is $L=0.15 \mathrm{~m}$, mean radius $R_{m}=0.0375 \mathrm{~m}$ and clearance $c=R_{b}-R_{j}=0.001 \mathrm{~m}$. Its mechanical properties are $E=205000 \mathrm{MPa}$ and $\nu_{b}=0.25$ (which correspond to a steel bearing) and its thickness is $2 \epsilon=3 \mathrm{~mm}$. The feeding pressure is $p_{a}=275000 \mathrm{~N} / \mathrm{m}^{2}$, the lubricant viscosity is $\nu_{f}=0.03882 \mathrm{~Pa} . \mathrm{s}$ and the velocity of the journal is $s=30 \mathrm{~m} / \mathrm{s}$. Finally, eccentricity (see Eq. (1.2)) is $\varrho=0.98$.

An analogous test was presented in [2], with slightly different boundary conditions: both elastic and hydrodynamic equations were posed in $\Omega$, a symmetry boundary condition being imposed on $\Gamma_{a}$. Neverthless, the mechanical interpretation remains still valid for the present work.

We can see in Figures 2 and 3 the pressure, concentration and relative displacement ${ }^{1}$ distributions obtained with a 1600 elements/900 nodes triangular mesh for the hydrodynamic subproblem, and a 3200 elements/1700 nodes triangular mesh for the elastic subproblem. First, we can appreciate the correlation between the highest pressure and the largest normal displacement (minimum gap region). Notice that we recover the symmetry of the normal displacement without imposing it, as was done in [2]. Moreover, due to convergentdivergent geometry, the pressure values decay to zero, which identifies the cavitation region, this one being also characterized by concentration values lower than one (see Eq. 1.4).

\section{THE COUPLED PROBLEM UNDER IMPOSED LOAD}

In previous works, the solution of some problems with imposed load were taken up; the elastic problem involved a plate model [14], or a Koiter shell model with a symmetry condition on the feeding groove [2]. As this kind of problems is more realistic from the mechanical engineering point of view, we show in this section the use of the new formulation, and the comparison with the results obtained in [2] with the symmetry condition.

\footnotetext{
${ }^{1}$ We call relative displacement the quotient $u_{3} /\left(h_{r}+u_{3}\right)$.
} 


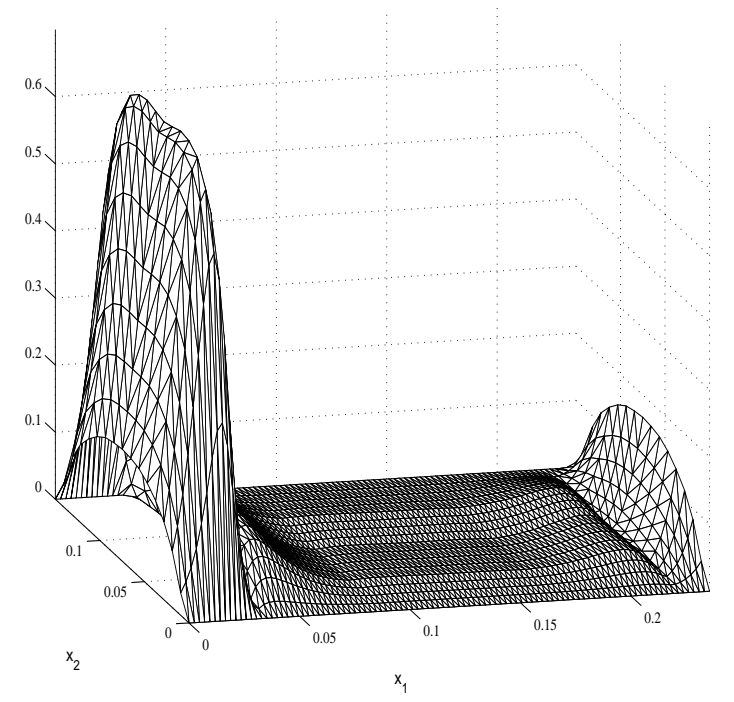

FiguRE 3. Relative displacement without imposed load.

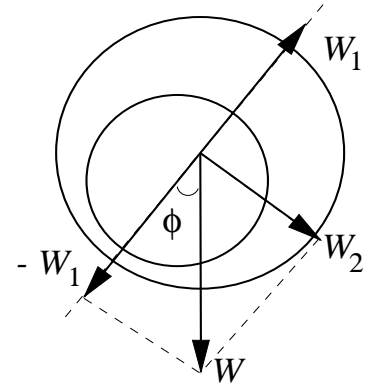

FiguRE 4. Load vector in the journal-bearing.

In the literature [10], the real gap between both cylinders is approximated by the expression

$$
h_{r}\left(x_{1}\right)=c\left[1+\varrho \cos \left(\frac{x_{1}-\phi}{R_{m}}\right)\right],
$$

the parameter $\phi \in[0,2 \pi]$ being the angle between the load vector and the line of centers (see Fig. 4).

In the imposed load problems framework, the additional unknowns $\varrho$ and $\phi$ have to be fitted by the additional load balance equations:

$$
\begin{aligned}
W^{2} & =\left(W_{1}^{*}\right)^{2}+\left(W_{2}^{*}\right)^{2} \\
\tan \phi & =-\frac{W_{2}^{*}}{W_{1}^{*}}
\end{aligned}
$$


TABLE 1. Comparison of two simulations.

\begin{tabular}{|c||c|c|c|c|c|}
\hline $\begin{array}{c}\text { Boundary } \\
\text { conditions }\end{array}$ & $\phi$ & $W^{*}$ & $\varrho$ & $\begin{array}{c}\text { Nr. Iter. } \\
\text { (load) }\end{array}$ & $\begin{array}{c}\text { Nr. Iter. } \\
\text { (pressure) }\end{array}$ \\
\hline Symmetry - Clamped & 22.2879 & 2482.61 & 0.9549 & 13 & 5261 \\
\hline Clamped - Clamped & 22.2916 & 2481.65 & 0.9549 & 13 & 5266 \\
\hline
\end{tabular}

where $\mathbf{W}=\left(W_{1}, W_{2}\right)$ is the given load vector, $W$ is its modulus and the components of the hydrodynamic load $\mathbf{W}^{*}$ are given by:

$$
\begin{aligned}
& W_{1}^{*}=\int_{\Omega} p\left(x_{1}, x_{2}\right) \cos \left(\frac{x_{1}-\phi}{R_{m}}\right) \\
& W_{2}^{*}=\int_{\Omega} p\left(x_{1}, x_{2}\right) \sin \left(\frac{x_{1}-\phi}{R_{m}}\right) .
\end{aligned}
$$

In [2], we had presented a complex numerical algorithm to solve the coupled Reynolds-Koiter problem and two numerical tests in order to validate its good performance. In both cases, we computed the pressure, concentration and normal displacements in a cylindrical journal-bearing device with circumferential feeding, clamped in both extremities. However, in order to reduce the time of calculus and taking into account the symmetry of the problem, we had meshed one half of the total domain, applying a symmetry boundary condition on $\Gamma_{a}$ for the elastic subproblem: displacement and normal rotation components were imposed to zero in the cylinder axis direction.

In the present work, we try to be as close as possible to the theoretical study we have developed in the previous sections, where both extremities are clamped. So, we have solved the problem described in Section 4.3, using the mesh of the total domain $\widetilde{\Omega}$ and the appropriate clamped boundary conditions for solving the elastic subproblem, and letting the algorithm iterate until the values of $\varrho$ and $\phi$ allow the hydrodynamic load to equilibrate the external load. A comparison between the two ways of resolution is summarized in Table 1.

In both cases, initial eccentricity, load modulus and load angle are $\varrho=0.98, W=2500$ and $\phi=30^{\circ}$, respectively. After comparing the good fitting between the approximated solutions obtained by both methods, the numerical results obtained with the clamped boundary conditions are shown in Figures 5 and 6 . Once again, the expected symmetry for the normal displacement is obtained.

\section{Conclusions}

The main goal of this paper is the proof of the existence of solution to a coupled problem that adequately models the elastohydrodynamic behaviour of a journal-bearing lubricated device. Although the proof follows the general scheme of previous works, as it is based on fixed point techniques, the coupling with an appropriate shell bearing model had never been treated. An additional and important difficulty arises from the fact that hydrodynamic and elastic equations are not written on the same domain.

This fact leads to a complex operator to be analyzed. Moreover, a numerical scheme is proposed to simulate a more realistic load imposed problem in a real data setting and the results agree with those presented in [2].

The present paper represents a first step to address a further theoretical study where thermal effects of load constraints are considered. To our knowledge, this kind of more complex coupled problems have not been theoretically treated in lubrication literature. 

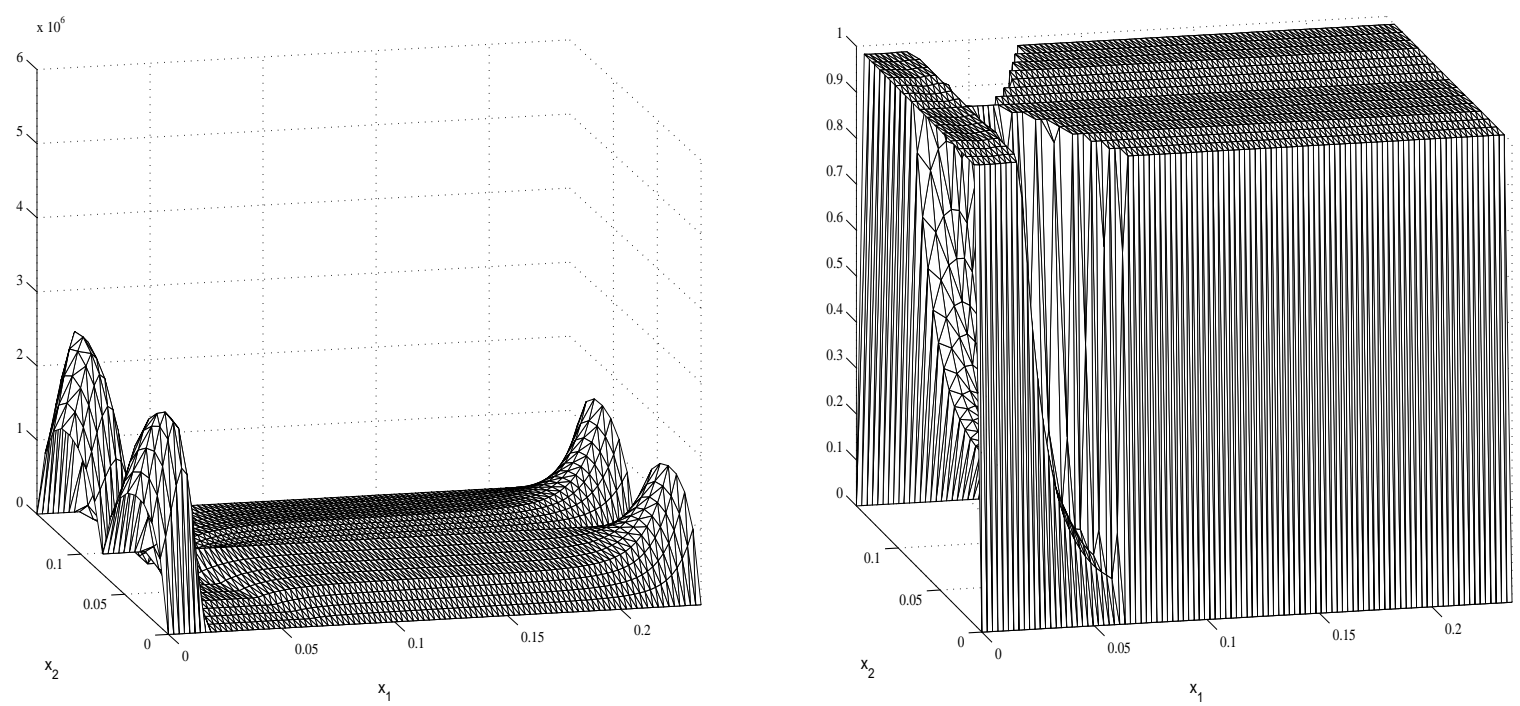

FiguRE 5. Pressure and concentration for the imposed load problem.

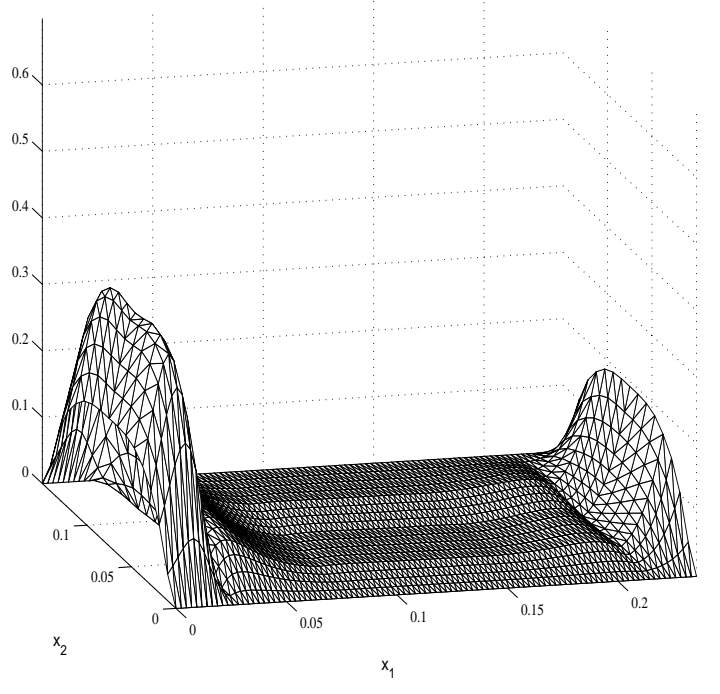

Figure 6. Relative displacement for the imposed load problem.

\section{REFERENCES}

[1] S. Alvarez, Problemas de frontera libre en teoría de lubricación. Ph.D. thesis, Universidad Complutense de Madrid (1986).

[2] I. Arregui and C. Vázquez, Finite element solution of a Reynolds-Koiter coupled problem for the elastic journal bearing. Comput. Methods Appl. Mech. Engrg. 190 (2001) 2051-2062.

[3] G. Bayada and M. Chambat, The transition between the Stokes equation and the Reynolds equation: A mathematical proof. Appl. Math. Optim. 14 (1986) 73-93.

[4] G. Bayada and M. Chambat, Sur quelques modélisations de la zone de cavitation en lubrification hydrodynamique. J. Theoret. Appl. Mech. 5 (1986) 703-729.

[5] G. Bayada, M. Chambat and C. Vázquez, Characteristics method for the formulation and computation of a free boundary cavitation problem. J. Comput. Appl. Math. 98 (1998) 191-212. 
[6] G. Bayada, J. Durany and C. Vázquez, Existence of solution for a lubrication problem in elastic journal bearing devices with thin bearing. Math. Methods Appl. Sci. 18 (1995) 255-266.

[7] M. Bernadou and P.G. Ciarlet, Sur l'ellipiticité du modèle linéaire de coques de W.T. Koiter. Lecture Notes in Appl. Sci. Engrg. 34 (1976) 89-136.

[8] M. Bernadou, P.G. Ciarlet and B. Miara, Existence theorems for two-dimensional linear shell theories. J. Elasticity 34 (1992) 645-667.

[9] H. Brézis, Analyse fonctionnelle. Masson, Paris (1983).

[10] A. Cameron, Basic lubrication theory. Ellis Horwood, West Sussex (1981).

[11] Ph. Destuynder, Modélisation des coques minces élastiques. Masson, Paris (1990).

[12] Ph. Destuynder and M. Salaün, A mixed finite element for shell model with free edge boundary conditions. Part I: The mixed variational formulation. Comput. Methods Appl. Mech. Engrg. 120 (1995) 195-217.

[13] Ph. Destuynder and M. Salaün, A mixed finite element for shell model with free edge boundary conditions. Part II: The numerical scheme. Comput. Methods Appl. Mech. Engrg. 120 (1995) 219-242.

[14] J. Durany, G. García and C. Vázquez, An elastohydrodynamic coupled problem between a piezoviscous Reynolds equation and a hinged plate model. RAIRO Modél. Math. Anal. Numér. 31 (1997) 495-516.

[15] J. Durany, G. García and C. Vázquez, Simulation of a lubricated Hertzian contact problem under imposed load. Finite Elem. Anal. Des. 38 (2002) 645-658.

[16] V. Girault and P.A. Raviart, Finite element aproximation of the Navier-Stokes equations. Lecture Notes in Math. 749, Springer (1997).

[17] T.G. Hughes, C.D. Elcoate and H.P. Evans, A novel method for integrating first- and second-order differential equations in elastohydrodynamic lubrication for the solution of smooth isotermal, line contact problems. Internat. J. Numer. Methods Engrg. 44 (1999) 1099-1113.

[18] D. Kinderlehrer and G. Stampacchia, An introduction to variational inequalities and their applications. SIAM, Philadelphia (2000).

[19] R. Verstappen, A simple numerical algorithm for elastohydrodynamic lubrication, based on a dynamic variation principle. $J$. Comput. Phys. 97 (1991) 460-488.

[20] S.R. Wu, A penalty formulation and numerical approximation of the Reynolds-Hertz problem of elastohydrodynamic lubrication. Internat. J. Engrg. Sci. 24 (1986) 1001-1013.

[21] S.R. Wu and J.T. Oden, A note on applications of adaptive finite elements to elastohydrodynamic lubrication problems. Comm. Appl. Numer. Methods 3 (1987) 485-494.

To access this journal online: www.edpsciences.org 